\title{
EL DESARROLLO DE LA INTELIGENCIA EMOCIONAL A TRAVÉS DE LA OPTIMIZACIÓN DE LAS CAPACIDADES COGNITIVAS A TRAVÉS DE LA EDUCACIÓN OBLIGATORIA
}

\author{
José Miguel Mestre Navas \\ Universidad de Cádiz \\ Nélida Pérez \\ Universidad de Alicante \\ Gabriel González de la Torre \\ Universidad de Cádiz \\ Juan Manuel Núñez Lozano \\ Centro de Apoyo a la Formación e Innovación Educativa de Irún, Gipuzkoa \\ Rocío Guil Bozal \\ Universidad de Cádiz
}

RESUMEN: Desde que la Inteligencia Emocional (IE) fue desarrollada en 1990 (Salovey y Mayer, 1990), era cuestión de tiempo que se desarrollaran aplicaciones como en la educación, entre otros. De hecho, proliferaron programas específicos de IE, de menor temporalidad y de complicada incardinación en los proyectos de centro. Sin embargo, hay una alternativa a realizarlo de forma inespecífica y de mayor duración y viabilidad. El presente trabajo se basa en una propuesta teórica como base para el desarrollo de la IE en la educación obligatoria. Partiendo del modelo cognitivo de habilidades mentales de Cattell-Horn-Carroll, se observa que las capacidades que son fomentadas en la educación obligatoria son las mismas que se observan en el segundo estrato del modelo; además trabajos recientes (véase Mestre, MacCann, Guil y Roberts, 2016) han señalado que dichas capacidades del segundo estrato están conectadas a las capacidades de la IE. Por consiguiente, toda actividad educativa que optimice la percepción, la comprensión, el control atencional o la planificación cognitiva está incidiendo en el desarrollo de la IE. Para ello, se insta a utilizar de forma transversal la introducción de información "hot" -aquella con significado existencial para un sujeto- en el contenido de las asignaturas (Mayer, Caruso y Salovey, 2016). Las asignaturas de ciencias y ciencias naturales favorecen el desarrollo de la percepción consciente y precisa, incluida de las emociones. Por su parte, las ciencias sociales y las humanidades fomentan la comprensión. Finalmente, las diferentes contingencias que se producen en la vida escolar son escenarios idóneos para el entrenamiento de la regulación de las emociones. 
PALABRAS CLAVE: Inteligencia Emocional, educación emocional, CattellHorn-Carroll Teoría de las capacidades mentales, aprendizaje socioemocional.

\title{
THE DEVELOPMENT OF EMOTIONAL INTELLIGENCE OPTIMIZING COGNITIVE ABILITIES THROUGH THE COMPULSORY EDUCATION
}

\begin{abstract}
Since Emotional Intelligence (EI) was developed in 1990 (Salovey \& Mayer, 1990), quickly the field of Education took advantage of the possibilities of El. Indeed, El-specific programs proliferated and developed as SEL-SocioEmotional Learning Programs. However, there is an alternative to realize nonspecifically and longer and viability way for improving the implicit abilities of El. Our document claims to work a theoretical proposal to develop the EI in compulsory education through the subjects. The proposal is based on the CattellHorn-Carroll Theory of Cognitive Abilities (CHC). CHC abilities are stimulated by the most of the mandatory subjects of both Primary and Secondary Schools. The second stratum of $\mathrm{CHC}$ model is composed of similar cognitive abilities as 1997 Ability El Model (perception, using, understanding, and managing emotions). Current works (see Mestre, MacCann, Guil, \& Roberts, 2016) have pointed out that these capacities of the second stratum are connected to the capabilities of El. Therefore, any educational activity that optimizes perception, understanding, cognitive attentional control or planning is affecting the development of El. Promoting El involves "hot" information processing. It is convenient to use transversely "hot information processing -this means that this information has special meaning for the people (Mayer, Caruso, \& Salovey, 2016). Connecting "hot" information to mandatory subjects teaching would develop the abilities of El. Sciences and Natural Sciences can improve emotional perception. However, Social Sciences and Humanities foster both emotional understanding and knowledge. Finally, the different contingencies that occur in school life scenarios are suitable for training of emotional regulation.
\end{abstract}

KEYWORDS: Emotional Intelligence, emotional education, Cattell-HornCarroll Theory of Cognitive Abilities, socio-emotional learning.

Recibido: 07/04/2016

Aceptado: 16/07/2016

Correspondencia: José Miguel Mestre Navas, Universidad de Cádiz, Facultad de Ciencias de la Educación, Campus Universitario del Río San Pedro, Avda. República Árabe Saharaui, s/n. 11510 Puerto Real (Cádiz) España. Email: josemi.mestre@uca.es.

\section{INTRODUCCIÓN}

El presente artículo trata de los aspectos cognitivos que deben potenciarse en la educación de la inteligencia emocional, estableciendo propuestas generales por etapas educativas. 
Se imaginan resolver un espacio-problema donde en vez de un analogismo verbal tuvieran que señalar la respuesta correcta a una situación emocional. Por ejemplo, de las siguientes opciones: ansiedad, tristeza, alegría, enfado o asco, ¿qué estado afectivo es más apropiado para una persona que se siente preocupada durante la mayor parte del tiempo? Basado en el conocimiento emocional, la respuesta más correcta sólo puede ser "ansiedad" (Mestre y Guil, 2012). La ansiedad es un estado emocional secundario que afecta a la percepción de vida existencial de una persona, cuyo proceso cognitivo central es estar preocupado (Lazarus y Lazarus, 1996). Otra cuestión inteligente, quizás más desafiante aún, es resolver la siguiente cuestión: ¿Cómo puede esta persona ansiosa regular efectivamente su estado? Daremos un valor pedagógico a lo lingüístico aquí: ¿Qué tal si quitamos el prefijo a "preocupación"? Así es, la manera más eficaz de regular los estados ansiosos es "ocuparse" de aquello que nos está afectando en lugar de "preocuparse" (Mestre, Araujo, Guil y Gil-Olarte, 2009). Este tipo de decisiones conlleva un acto inteligente (Salovey, Hsee y Mayer, 1993) porque usamos la información proveniente de las emociones para que nos ayuden a tomar decisiones más efectivas (Kahneman, 2011) siempre y cuando utilicemos el sistema cognitivo controlado y no el automático -del que hablaremos después-.

De este tipo de cuestiones trata, o deberían tratarse, cuando hablamos de Inteligencia Emocional (IE). Es decir, de cómo resolver problemas cuando la información que usamos provienen de las emociones. En 1990, Salovey y Mayer propusieron la existencia de esta nueva inteligencia basándose en la investigación de diferentes áreas como la cognición, la emoción, la inteligencia o la psicoterapia, sugiriendo que algunas personas podrían ser más inteligentes con las emociones que otras (Salovey y Mayer, 1990). Posteriormente, señalaron qué capacidades cognitivas consideraban más importantes sobre su constructo y desarrollaron un modelo jerárquico que de abajo a arriba viene descrito como: percibir, usar, comprender y gestionar las emociones en uno mismo y en los demás (Mayer y Salovey, 2007, en castellano). El nivel más bajo "percepción" se desarrolla antes que el último "regulación" (Mestre, 2003). Algunos autores, no terminan de ver la facilitación como una capacidad independiente de las otras tres, sino que la denominada segunda rama queda integrada dentro de las otras tres. Por lo que la IE tendría tres capacidades: percepción, comprensión y regulación -en vez de gestión de emociones que parece tener un matiz menos cognitivo que regulación (Mestre, MacCann, Guil y Roberts, 2016; Mikolajczak, 2009)-. Será importante atenernos a estas tres (percepción, comprensión y regulación) cuando queramos explicar cómo fomentar la IE.

\section{Principios de la Inteligencia Emocional}

Recientemente, con motivo del $25^{\circ}$ aniversario del constructo, Mayer, Caruso y Salovey (2016) han establecido siete principios sobre el que la IE debe ser atendida y comprendida. El primero no deja lugar a dudas de qué es la IE: una capacidad mental. Cuestión obvia si tenemos en cuenta que el término IE conlleva el sustantivo "inteligencia" y el adjetivo "emocional". Sin embargo, algunas posturas como los denominados modelos de rasgos sostienen que el término incluye ciertos rasgos de personalidad (Mestre y Fernández-Berrocal, 2007). Nosotros optamos por una 
visión de la IE como un constructo de la inteligencia, si bien en un sentido más amplio que la tradición psicometricista de la misma (Mestre, 2003). Si la inteligencia es la capacidad para llevar a cabo razonamiento abstracto, comprender significado, saber distinguir entre similitudes y diferencias entre dos conceptos, generalizar situaciones a partir de hechos y comprender cuándo se debe aplicar excepciones, entonces este sistema de capacidades mentales (Detterman, 1982) pueden también aplicarse a las emociones para identificar quienes son capaces de percibir emociones de forma precisa, usarlas para facilitar el pensamiento, comprender las emociones y su significado y, con ello, gestionar las emociones en ellos mismos y otros (Mayer et al., 2016).

El segundo principio es que la IE está mejor medida como una capacidad que como una escala o autoinforme. Como cualquier instrumento de evaluación de la inteligencia, la IE debiera estar medida mediante tareas cognitivas -denominadas performance measures (Mestre y Guil, 2006)-. Es difícil justificar por qué la IE podría ser medida de una forma diferente a otras inteligencias; cuando ya se observan cómo los datos de IE (como capacidad) se estructuran de forma similar a las definiciones actuales de inteligencia (MacCann, Joseph, Newman y Roberts, 2014). Además, la gente es bastante inexacta estimando sus propias capacidades tanto de inteligencia como de IE (Brackett, Rivers, Shiffman, Lerner y Salovey, 2006), ¿por qué habríamos de estar seguros de dichas autoestimaciones? (Mestre y Guil, 2006). ¿Tiene sentido preguntar cómo de inteligente somos en una escala de 1 a 5 ? Solamente en el caso de que nos interese conocer la percepción que las personas tienen de su nivel de inteligencia.

El tercer principio es que resolver de forma inteligente problemas no necesariamente se corresponde con comportamientos inteligentes. No hay nada extraño en esta aseveración y lo explicaremos con un ejemplo. Si un niño/a está dotado/a para el rendimiento cognitivo, ¿ello implica necesariamente que tenga un buen rendimiento académico? Todos conocemos casos de estudiantes cognitivamente dotados con un pobre rendimiento académico (por numerosas causas que no viene al caso relatar ahora). Es más, el hecho de tener un rendimiento académico por debajo de lo esperado podría ser definido como un acto poco inteligente, pero ello no le priva a ese niño/a de estar sobradamente cognitivamente capacitado/a. La IE no es diferente de las otras inteligencias, en esto tampoco (Duckworth, Quinn y Tsukayama, 2012). Por ejemplo, algunos adolescentes varones con elevada IE son socialmente poco valorados por sus compañeros y profesores, sin embargo demuestran una buena capacidad en pos del logro académico y de cierto nivel resiliencia educativa (Lopes, Mestre, Guil, Kremenitzer y Salovey, 2012). Es curioso que este patrón de elevada IE se mantiene, sólo con ciertos varones, a lo largo de la etapa de secundaria pero no con las mujeres que prefieren usar su IE para mantener una buena valoración social de sus compañeros (Mestre, Guil, Lopes, Salovey y Gil-Olarte, 2006). Es decir, la predicción teóricamente previsible de buena aceptación social no siempre se cumple en personas con elevada IE, al menos con un elevado porcentaje de varones de secundaria con un buen rendimiento en un test de tarea cognitiva como el MSCEIT (Mayer, Salovey, Caruso Emotional Intelligence Test, Mayer, Salovey, Caruso y Sitarenios, 2003). No olvidemos que el término "inteligente" es un adjetivo, mientras que "inteligencia" es un sustantivo. Las connotaciones lingüísticas son 
importantes para no perdernos en vaguedades terminológicas. El comportamiento emocionalmente inteligente está más relacionado con el constructo de personalidad que con el de inteligencia (Mayer et al., 2016) aunque se manifiesten correlaciones significativas entre bajas y muy bajas (Joseph y Newman, 2010).

Los dos siguientes principios tienen relación en cómo debe ser medida la IE. El cuarto, el contenido del test de IE a aplicar debe cubrir el área del problema a resolver. Por regla general, los test de inteligencia son largos en número de ítems y en tiempo, aproximadamente unos 45 minutos en adultos, de realización porque cubrir la esfera de las capacidades incluidas en la inteligencia así lo requiere, medir la IE como capacidad no va estar exenta de dichos parámetros (Nafukho, 2010). A mayor edad, mayor complejidad de los elementos a incluir en una prueba de IE, como ocurre con otros tipos de inteligencia el nivel de una prueba de inteligencia sube con la edad, y eso mismo ocurre con la IE (Mestre, Núñez-Vázquez y Guil, 2007). En la etapa infantil, cuando la teoría de la mente es incipiente en ellos, no podemos plantear cuestiones que impliquen un elevado conocimiento del lenguaje, o un claro desarrollo cognitivo para entender emociones más complejas como la envidia, o una situación elaborada de un conflicto emocional a resolver que le exijan una memoria operativa más allá de lo que de su edad aún le permite. Una prueba más apropiada sería saber identificar emociones según una etiqueta o realizar valoraciones breves de comparación (del tipo quien está más triste en esta imagen) (Mestre, Pérez-Alarcón, Larrán, Guil y Hidalgo, 2014). El Quinto principio es que estos tests de IE representan una puntuación de cómo la capacidad de las personas, y sus diferencias en puntuación, reflejan a su vez su habilidad mental para solucionar problemas de contenido emocional. Por lo que si el test abarca ítems que reflejan pobremente el contenido de la IE, entonces dichos tests no representan correctamente bien aquello que se quiere medir y podríamos hacer previsiones erróneas (Mayer et al., 2016).

El sexto, es que la IE es una inteligencia amplia. Es decir, la IE queda mejor encuadrada en aquellas perspectivas que poseen una visión "molar" de la inteligencia mejor que una "molecular" -como la perspectiva factorialista de la inteligencia-. Por tanto, la IE queda teóricamente mejor encuadrada en la perspectiva adaptativa de la inteligencia (Mestre, 2003). La perspectiva adaptativa de la inteligencia viene representada por autores como Gardner, Sternberg o antecedentes anteriores como Vygotsky o Piaget, o de filósofos como Platón o Aristóteles (Plucker y Esping, 2014). Esta perspectiva defiende que la inteligencia se mueve entre capacidades amplias y estrechas. En concreto, el modelo de inteligencia más influyente en la actualidad es el Cattell-Horn-Carroll (CHC) que durante un siglo ha ido asentándose en la literatura especializada, también es conocido como el modelo de los tres estratos (Schneider y Newman, 2015) y tiene una visión amplia o molar de la inteligencia. El modelo CHC será de gran interés para nosotros para hacer entender al lector cómo podríamos fomentar las capacidades cognitivas implícitas en la IE. El modelo $\mathrm{CHC}$ (ver figura 1) en su estrato 3 tiene el factor $g$ que es una capacidad general que se relaciona con las demás, como si fuese el "pentium" o acelerador de las otras capacidades.

Lo interesante viene en el segundo estrato, un conjunto de capacidades más amplias que las del primer estrato, donde algunos trabajos ya han señalado que la 


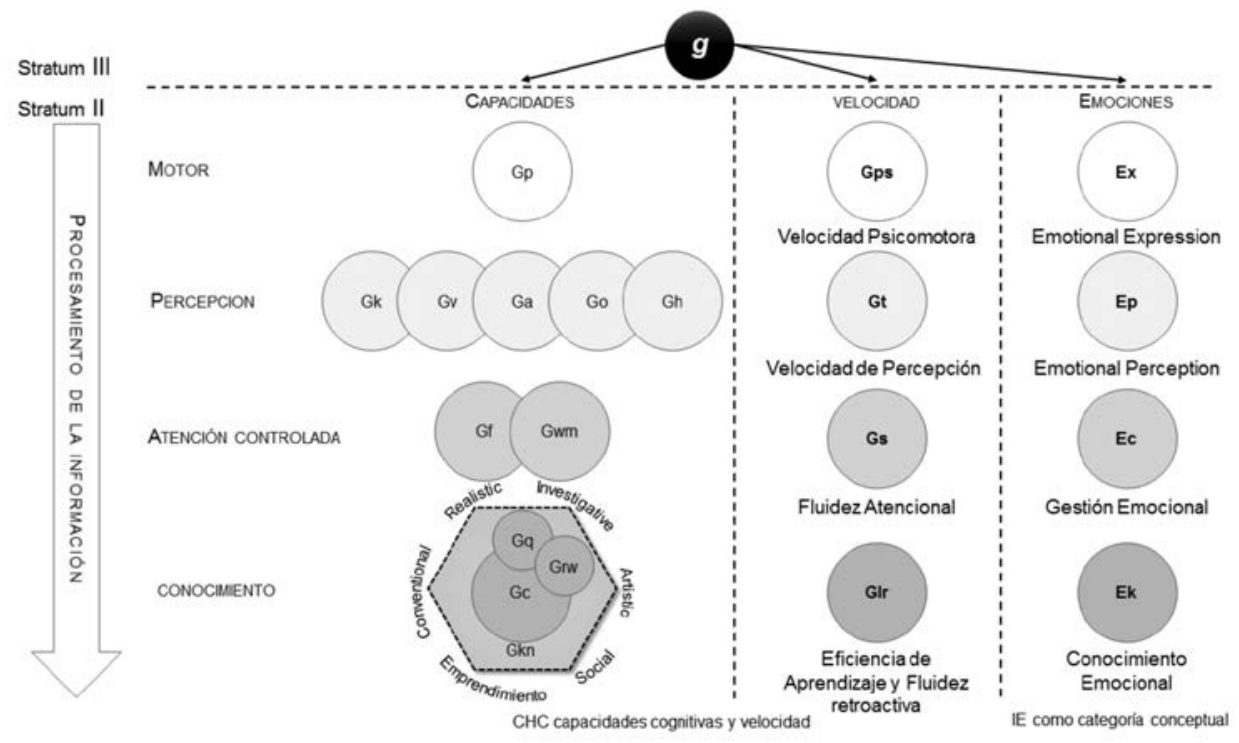

Figura 1. Cattell-Horn-Carroll (CHC) Teoría de las Capacidades Cognitivas con la Inteligencia Emocional (IE) como una categoría conceptual

Una representación de la CHC Theory of Cognitive Abilities en el Segundo estrato unido a la IE como una categoría conceptual. Cada nivel determina el poder de capacidad del procesamiento de la información (motor, perceptivo, atención controlada y conocimiento) y la velocidad de cada una está relacionada como una categoría conceptual. La Teoría Cognitiva CHC tiene tres estratos: el primero no es representado en la figura.

Leyendas: A nivel motor: Gp representa a las Capacidades Psicomotoras. En el nivel de Percepción: Gk es Kinestésia, Vb Capacidad Visoespacial, Ga Audición, Go Olfato y Gh Tacto. Atención Controlada: Gf es Razonamiento Fluido y Gwm es Memoria de trabajo. Nivel de Conocimiento: Gc Comprensión Verbal y Conocimiento, Gq Conocimiento Cuantitativo, Grw Lectura/Escritura y Gkn Conocimiento de un Dominio Específico. Basado en Schenide y Newman (2015).

IE parece estar conectada también con estas capacidades del segundo estrato (MacCann et al., 2014) y, por consiguiente, las capacidades amplias que se describen en el modelo CHC (véase Flanagan y Dixon, 2013; McGrew, 2009) también pueden ser encontradas en las de la IE: Percepción, comprensión y regulación de las emociones (MacCann et al., 2014; Mestre et al., 2016) tal y como refleja la figura 2. Si bien en el reanálisis de los datos otros autores encontraron los cuatro componentes de la IE en el Segundo estrato del modelo CHC (Legree et al., 2014). Resumiendo, las capacidades del Segundo estrato del modelo $\mathrm{CHC}$ conectadas con la IE son aquellas que teóricamente, necesitamos desarrollar y estimular ante contenidos o desafíos que tengan a la emoción como protagonista. Por lo que nuestra tesis es que al estimular estas capacidades, también estimulamos de forma directa o no la IE. Como veremos después.

El séptimo, y último principio de Mayer et al. (2016), es que la IE está centrada en el procesamiento de información implicada con la información que tiene especial significado para los individuos (de hecho, la llaman "hot information processing"). 


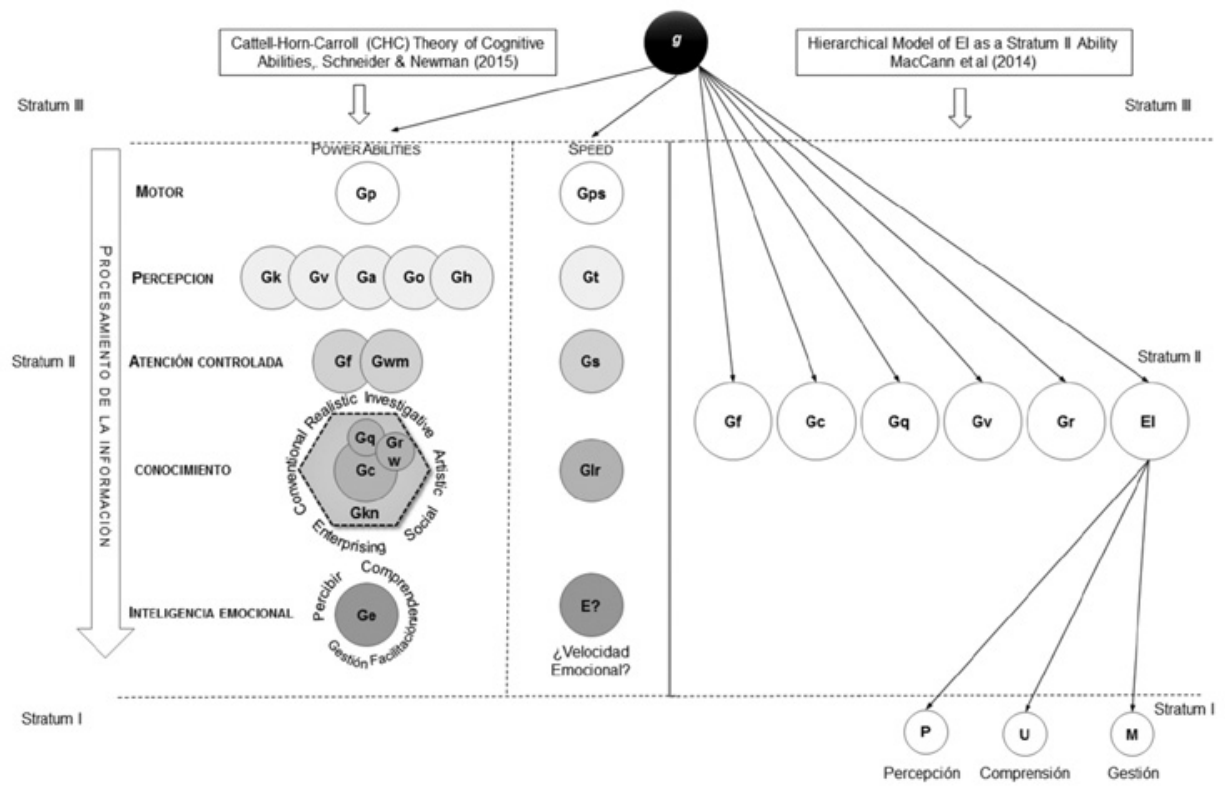

Figura 2. Cattell-Horn-Carroll (CHC) Teoría de las Capacidades Cognitivas vs. Modelo Jerárquico de la Inteligencia Emocional en el Estrato II

Una representación de la Teoría CHC de Capacidades Cognitivas en el Estrato II (Schneider y Newman, 2015), el cual está relacionado con el modelo jerárquico de la IE como capacidad en el Estrato II. Según MacCann et al. (2014), la IE pertenece al Segundo estrato y tres de sus ramas, quitando la facilitación, estarían incluidas en el Estrato I, denominándose modelo PUM de la IE (Perception, Understanding, and Managing, PUM). En la Fig. 1 están descritas las leyendas para las capacidades.

Para Mayer et al. (2016) dividen la información procesada en "hot" y "cool". La información "cool", o fría, es aquella relacionada con hechos o significados sin valor personal para el sujeto como el cálculo de una ecuación matemática. Sin embargo, la información "hot", o caliente, es toda aquella por la que los individuos tienen importancia para ellos: la aceptación social, la identidad coherente, o el bienestar emocional. A diferencia de las otras inteligencias, la IE lidia con la información "hot", la que tiene un valor y un significado adaptativo, que no existencial, para las personas. Y este principio es importante para el desarrollo de la IE, toda actividad mental que conecte la capacidad cognitiva con este tipo de información es, teóricamente, un acto de inteligencia emocional. La emoción tiene una función fundamental: la adaptación (Palmero y Mestre, 2004). Así que la IE es un conjunto de capacidades cognitivas, fundamentalmente percibir, comprender y regular las emociones, dedicadas al tratamiento de la información "hot" y cuyo propósito es la adaptación tanto personal como social. El acto inteligente es señalado por Mayer et al. (2016) con el adjetivo "precisión". Es decir, percibir, usar, comprender y regular la emoción con precisión.

Visto el marco teórico, ¿cómo podríamos Ilevarlo a cabo sin que ello implique una revolución en los proyectos curriculares de los centros escolares? 


\section{LA RELACión ENTRE LA INTELIGENCIA EMOCIONAL Y CRITERIOS DE FUNCIONAMIENTO ADAPTATIVO EN CONTEXTOS EDUCATIVOS}

Partiendo de la idea que tenemos de que la IE debe ser entendida como capacidad, contamos en la actualidad con suficientes evidencias empíricas para considerar que la IE podría ser una capacidad susceptible de ser enseñada en contextos educativos.

Según un detallado meta-análisis donde se muestran los resultados que integran un sistemático proceso de programas de desarrollo socioemocional (conocidos como SEL de "socioemotional learning"), la implementación de estos programas incrementa el éxito académico, mejoras en la relación entre el alumnado y el profesorado y el descenso en las conductas disruptivas del alumnado (Durlak, Weissberg, Dymnicki, Taylor y Schellinger, 2011). De forma general, los "SEL's" se refieren a los procesos implicados en el desarrollo del concimiento emocional y de la regulación de las emociones en uno mismo y en los demás que mejoran las interrelaciones y las tomas de decisiones socialmente deseables (Durlak, Domitrovich, Weissberg y Gullotta, 2015).

El interés del entrenamiento de la IE, bajo este paraguas del SEL, es que el desarrollo de estas competencias parece crítico para el bienestar, las relaciones de calidad, el rendimiento académico y la adaptación escolar (Levin, 2012; Nathanson, Rivers, Flynn y Brackett, 2016). Esta estrecha relación entre el entrenamiento de la IE y estos beneficios socioemocionales suponen una buena inversión social y educativa (Belfield et al., 2015). No obstante, es necesario resaltar que los propios autores más volcados con el entrenamiento de las capacidades de la IE aún demandan la necesidad de dar viabilidad a la enseñanza de estas habilidades en contextos educativos (Brackett y Rivers, 2013; Nathason et al., 2016). Esta demanda viene dada bien por la inclusión del entrenamiento de habilidades específicas dentro del proyecto curricular de centro o bien por su entrenamiento inespecífico. Es decir, por desarrollar dichas capacidades de una forma integrada y transversal dentro del plan de estudios del centro escolar y no como un programa adyacente y externo dentro del curriculum. Si el centro tiene interés por un programa externo hay varios programas y experiencias SEL, como el RULER (Nathason et al., 2016), o en España concretamente el INTEMO para adolescentes (Ruiz Aranda et al., 2013) o propuestas avaladas para la etapa infantil como el "braini emotions" (Pérez, Figueras, Mestre, Moltó y Guillén, 2016).

Sin embargo, nosotros proponemos otra alternativa. Enseñar la IE de una forma menos específica, más prolongada en el tiempo, integrada de forma transversal en el currículo del centro y centrado en el desarrollo de cualquier capacidad cognitiva que pueda generalizarse a una situación emocional. Es un debate, si es que lo llegase a haber, parecido a los programas de prevención de drogas en los centros escolares. Durante un tiempo se realizaron programas específicos de prevención drogas en los centros; hasta que las evaluaciones de los programas advirtieron que era más eficaz realizarlo de forma específica. Es decir, por ejemplo, es inviable el consumo de drogas con la realización de actividades incompatibles como el deporte (Rementería, 2001). ¿Podría ser más viable y realista un programa "no específico de IE" a largo plazo que un programa específico de IE a corto plazo? Hipotetizamos que así podría ser. 
¿Qué es más efectivo en el tiempo, un programa de IE de 15 días o incorporar el contenido emocional en diversas asignaturas que impliquen añadir información "hot" en el desarrollo de las capacidades cognitivas? ¿Pueden ser generalizables las competencias cognitivas que implica comprender textos con el desarrollo de la IE? ¿Qué podría ser más interesante para un centro, que el profesorado aprenda, además de muchas otras demandas sociales, a ser inteligente emocional o realizar su trabajo como habitualmente, pero incorporando contenidos emocionales en muchas de las asignaturas? Un análisis detallado a los trabajos del SEL en contextos educativos y se podrá observar que en dichos centros no suelen ser públicos sino privados; y que requieren de un esfuerzo extra en recursos económicos y humanos. Esta perspectiva que presentamos no anula en absoluto las iniciativas específicas, simplemente es una alternativa a ellas que los centros pueden acogerse en caso de desear estimular las capacidades descritas en la IE. Incluso lo más pertinente sería mantener ambas perspectivas de enseñar a IE; pues a mayor número de oportunidades de aprendizaje, mayor sería la probabilidad de aprender dichas capacidades.

Por ejemplo, Ivcevic y Brackett (2015) señalaron que cuando explícitamente se enseña IE también se potencia la capacidad del alumnado para resolver problemas complejos y reales de la vida diaria. ¿Acaso no ocurriría también de forma viceversa? Si enseñamos a los estudiantes a prestar atención durante la resolución de un problema de principio a fin monitoreándolo, identificar correctamente lo que el problema demanda y elegir qué estrategia sería la más precisa para resolver dicho problema, por ejemplo, en una asignatura como las ciencias naturales, ¿estaríamos en disposición de generalizar estas capacidades con las situaciones emocionales? Nosotros creemos que sí.

\section{Del cómo con el desarrollo de las capacidades cognitivas en ALGUNAS ASIGNATURAS PUEDE SER GeNeRALIZADAS AL DESARROLlO DE LA INTELIGENCIA EMOCIONAL}

\section{Percepción}

Todo proceso cognitivo empieza y termina con la Percepción. En el caso que atendemos aquí, percibir emociones es la capacidad por la que se empieza a construir la IE. Como ya comentamos, la primera capacidad descrita en el modelo de capacidad de la IE es "percibir de forma precisa las emociones" (Mayer y Salovey, 2007). Sin embargo, con decir "precisa" no basta. Porque la percepción es un doble proceso, mayormente simultáneo, de los denominados procesos "de arriba abajo" y "de abajo arriba" (top-down vs. bottom-up, Kosslyn y Miller, 2013). Según Galotti (2008), los procesos de abajo a arriba ocurren cuando interpretamos en función de los que percibimos de los datos. Es decir, nos atenemos estrictamente a lo que nuestros órganos sensoriales perciben. Esto implica un mayor esfuerzo mental y no estar mediatizado por filtros "automáticos" como las creencias, los estereotipos o los prejuicios (Mestre, Comunian y Comunian, 2007). Algo similar a lo que busca el mindfulness percibir sin valorar, "limpiar el filtro cognitivo de la turbiedad de las ideas preconcebidas" (Márquez y Mestre, 2012). Por consiguiente, percibir emociones de forma precisa debe ser mayormente un proceso perceptivo "de arriba 
abajo" (Mestre, Larrán, Herrero, Guil y de la Torre, 2015) porque debería reflejar "precisión" a la hora de identificar las expresiones emocionales de los demás y las propias. Consecuentemente, es más eficaz identificar las emociones por lo que nuestros sentidos nos reportan que por lo que nos reportan nuestras expectativas (que sería un proceso de "arriba a abajo").

Sin embargo, en casi todas las situaciones, el conocimiento previo o las expectativas están implicadas en la percepción emocional (Mestre et al., 2015). Este proceso denominado "de arriba abajo" abarca las capacidades mentales implicadas en dirigir tanto la observación como los estímulos externos hacia unas ideas previas adquiridas de una exploración ya adquirida (Goldstein, 2008). Este proceso es más rápido, de menor esfuerzo y ayuda al ahorro de energía mental. Sin embargo, es "altamente perezoso" y muy susceptible de cometer errores (Kahneman, 2011). No obstante, las personas perciben más allá de lo que la mera información proporciona pues nos afecta el aprendizaje previo (Bruner, 1973).

Kahneman (2011), como señalamos al principio del documento, describe que el funcionamiento cognitivo, como también ocurre con el emocional, posee dos sistemas. El Sistema 1 opera de manera rápida y automática, con poco o ningún esfuerzo y sin sensación de control voluntario. El Sistema2 centra la atención en las actividades mentales desafiantes que lo demandan, por ejemplo, los cálculos complejos. Las operaciones del Sistema2 están a menudo asociadas a la experiencia subjetiva de actuar, elegir y concentrarse. Y ambos sistemas pueden trabajar de forma simultánea, como de hecho así suele hacerse (Mestre, Comunian et al., 2007).

Sin embargo, este sistema 2 implica el uso de la atención para que dicha actividad cognitiva no quede perturbada. Veámoslo con algunos ejemplos: estar atento al planteamiento de un problema de matemáticas, concentrar la atención en una explicación dada, escuchar una respuesta en medio de varias conversaciones, buscar la respuesta correcta en un examen tipo test, observar un comportamiento adecuado en una situación social, resolver un conflicto, contar las veces que un fenómeno ocurre, comparar y decidir qué opción es mejor, y así podríamos seguir. Pero obsérvese, que todas estas acciones requieren de atención, concentración, planificación cognitiva, monitorización, comprensión y gestión de la información disponible. De la misma manera que la IE necesita del control atencional para el desarrollo de una acción que le permita una solución exitosa en un conflicto emocional (Mestre et al., 2016).

Sin el papel activo de la atención estas actividades se realizan mal o no se realizan en absoluto (Kahneman, 2011). Y la atención está ligada a la noción de esfuerzo. Es probable que el profesorado de primaria y secundaria identifique estas acciones cognitivas en sus asignaturas correspondientes. Especialmente los de ciencias y los de ciencias naturales. Así que, al estimular estas actividades escolares, retroalimentarlas y generalizarlas a situaciones de la vida cotidiana donde las emociones están presentes es, desde nuestro punto de vista, también una forma de alimentar las habilidades y capacidades cognitivas implícitas en la IE.

Por consiguiente, la diferencia entre malinterpretar, o sobreinterpretar, y percibir de forma precisa una situación emocional depende mucho de no dejarse influir por las ideas previas, las creencias irracionales o los prejuicios. Medina 
et al. (2012) realizaron una interesante investigación donde se comparaban los resultados de la IE (medida con el MSCEIT) con el de sensibilidad social (medida con el TESIS, Barraca, Fernández-González y Sueiro, 2009). Los resultados mostraron que las personas con menos puntuación en IE también fueron aquéllas que obtuvieron una mayor puntuación en la escala de sobreinterpretación del TESIS. Sin embargo, sensibilidad social que implica precisión en los juicios las relaciones fueron positivas con la IE. Por consiguiente, la IE ayuda de alguna manera a no utilizar sesgos erróneos en nuestros razonamientos sobre situaciones sociales con emociones presentes.

Cuantas más oportunidades ofrezca el profesorado situaciones que estimulen al alumnado a percibir correctamente, a mejorar la atención y monitorizar de principio a fin el proceso de planificación cognitiva, más posibilidades de aprendizaje de IE también le estamos ofreciendo. Si se facilitasen situaciones donde las emociones están presentes, como un conflicto en el aula, ¿por qué no puede un profesor de matemáticas, por ejemplo, afrontarla como un problema de matemáticas? No es una cuestión banal esta, las emociones conllevan informaciones adscritas -por ejemplo, tras la tristeza se sustenta la valoración de pérdida. Percibir, comprender y gestionar la tristeza requiere de las mismas capacidades cognitivas que resolver un problema de química. Percibir, entender y planificar cognitivamente con las emociones conlleva comprender que las emociones tienen funciones adaptativas, homoestáticas y sociales en nuestra vida personal e interpersonal y que son parte de una "ecuación" (Mestre, Guil, Brackett y Salovey, 2008).

\section{Comprender las emociones}

Comprender emociones es una capacidad que está muy ligada a la inteligencia cristalizada (Mestre et al., 2016). El acúmulo de conocimiento a lo largo de la vida de una persona incide, cómo no, en el desarrollo de esta capacidad para comprender las emociones es considerada por algunos "como el corazón de la regulación inteligente" (Wranik, Barrett y Salovey, 2007, p. 395). Comprender cómo las Emociones se combinan, progresan y cambian a través del tiempo y las situaciones (Mayer y Salovey, 2007) requiere de un largo aprendizaje e instrucción, hasta tal punto que el desarrollo evolutivo y adecuado de la IE requiere de un apropiado desarrollo cognitivo, social, moral, emocional y lingüístico (Mestre, Núñez-Vázquez et al., 2007). Comprender las emociones sociales (culpa, vergüenza, celos o ansiedad, por ejemplo) requiere más tiempo que las emociones básicas (alegría, tristeza o ira, por ejemplo).

Desde nuestro punto de vista, ahora son las asignaturas vinculadas a las ciencias sociales las que más pueden aportar al conocimiento de las Emociones. Y la literatura es una de ellas. La obra de Otelo de Shakespeare es una oportunidad para entender las diferencias y las conexiones entre los celos y la envidia. Su trama es compleja, pero entender el papel de las emociones de los personajes de Yago y Otelo ayudan a comprender las diferencias entre celos y envidia.

Independientemente de la precisión de los hechos, comprender las motivaciones de los personajes requiere entender por qué Otelo siente celos y Yago envidia de Otelo. El "moro" Otelo es un general al servicio de Venecia que ha conquistado el amor 
de Desdémona, hija del senador Brabantio quien acusa a Otelo, ante el Dux de Venecia, de haberla hechizado y raptado a su hija. Pero Desdémona confirma cómo Otelo conquistó lealmente su corazón. Ante un inminente ataque de los turcos a Chipre, Otelo es requerido para resolver la amenaza, y Brabantio de mala gana cede y accede a que su hija Desdémona le acompañe a Chipre. Por su parte, el alférez Yago ha sido sustituido por Casio, siente un profundo odio por Otelo, quien ha oído rumores de su esposa Emilia, y camarera de Desdémona, ha yacido con Otelo. Yago envidia a Casio, y logra desacreditarle tras hacer que se embriague y turbe la paz pública. Tras verse privado de su grado, el "envidioso" Yago induce a Casio a que ruegue a Desdémona que interceda en favor suyo; simultáneamente Yago hace nacer en el ánimo de Otelo la sospecha de que su esposa le engaña con Casio. La "maquiavélica" acción de Yago surte efecto cuando Desdémona intercede ante Otelo en favor de Casio. Lo que provoca en Otelo la confirmación de las sospechas inducidas por Yago y crea en él los sentimientos de celos. El "envidioso" Yago se las ingenia para que una prenda que Otelo regaló a Desdémona sea hallada en poder de Casio. Otelo, cegado por los celos, ahoga a Desdémona en su lecho. Finalmente, la trama de Yago es descubierta y Otelo se suicida estoicamente.

A diferencia del inglés, el castellano deja más claro las diferencias entre celos y envidia. Donde en inglés parece prácticamente una diferencia de intensidad con algunos matices claves; en español celos y envidia tienen acepciones cualitativamente diferentes (Smith, Kim y Parrot, 1988). La envidia es una emoción en la que intervienen dos personas, sin embargo, en los celos son tres, en lo que la persona celosa se siente amenazada por la creencia de que su pareja siente algo por una tercera persona (Salovey, 1991). Entender la transición entre emociones y las causas y relaciones de una obra literaria ayuda indudablemente a ir comprendiendo las emociones complejas. El estado subjetivo de una persona envidiosa es querer lo que otro tiene, y en una actitud hostil llegar a desear que la otra persona pierda lo que el envidioso anhela (Lazarus y Lazarus, 1994).

La razón de por qué hemos elegido la trama de Otelo es para señalar que tenemos asignaturas, sobre todo de ciencias sociales, donde el alumnado puede ser indagado o mayéuticamente interrogado y estimulado para ir encontrando respuestas y conocimiento sobre emociones, especialmente las complejas que requieren de una mayor exigencia cognitiva que las básicas. En concreto si fomentamos las humanidades e incluimos información "hot" como tramas emocionales, también estamos fomentando la comprensión de las emociones. La historia o la literatura, e incluso la religión o la ética, son susceptibles de ser transversalmente analizadas desde las emociones. Por ejemplo, el Tratado de Versalles por el que se pone fin a la primera guerra mundial fue percibido como humillante por los alemanes, que desencadenó en cierto resentimiento, junto con el crack económico de 1929, fue utilizado por Hitler y sus seguidores para estimular el exagerado sentimiento "ario" o hubris (orgullo desmedido) germánico que facilitó la segunda guerra mundial entre otras razones combinadas (Rodrigues-Gonzales, 1995).

Estamos seguros de que añadir motivaciones y emociones aumentaría el interés del alumnado por las ciencias sociales, produciéndose una simbiosis mutua entre la mejora del conocimiento adquirido y el desarrollo de la comprensión de las 
emociones. Es curioso observar que cuando en 1997 Mayer y Salovey (véase en 2007 en castellano) describieron pormenorizadamente la IE, utilizaron una situación escolar que generó en conflicto utilizando la redacción del alumnado como una expresión de la IE para resolverlo. Cuando un adolescente redacta utiliza varias estrategias cognitivas: debe poner atención y concentración en la tarea de analizar cuidadosamente cuál es el problema, aportar las razones del porqué de dicho problema y dar una visión razonada de cómo solucionarlo. Todas estas estrategias cognitivas son capacidades necesarias para un buen desarrollo de la IE (Mayer y Salovey, 2007).

\section{Regular las emociones}

La regulación de emociones se centra en el proceso de cómo la gente usa ciertas capacidades (valoración y atención, fundamentalmente) para influir en qué emociones se tienen, cuando se tienen y cómo tienen que ser expresadas (Gross y Thompson, 2007). Para Gross y Barret (2011), desde una perspectiva pragmática del concepto de regulación de emociones $(\mathrm{RdE})$, describen la $\mathrm{RdE}$ como una transacción donde hay una situación dada que implica la atención de una persona hacia una meta relevante, la cual activa en el sujeto tanto la interpretación (un proceso de arriba a abajo, Mestre y Guil, 2012) de tales elementos como su respuesta cognitiva, comportamental como fisológica a dicha situación. Recientemente, Peña-Sarrionandia, Mikolajczak y Gross (2015) sugirieron, mediante un meta-análisis, integrar, de forma simultánea, la regulación de emociones y la IE para entender dos cosas: cómo desde las diferencias individuales se gestiona mejor o peor las emociones y cómo es el proceso de regular emociones. Las personas con una elevada IE usan estrategias como la revaloración positiva y la modificación directa, sin embargo, aquéllos con una baja puntuación en IE tienden a usar más la evitación y el pensamiento rumiativo (Peña-Sarriondia et al., 2015). Es decir, a tenor de los resultados del meta-análisis, las personas con una buena IE parecen utilizar un mecanismo para traducir su conocimiento emocional sobre la IE para realizar comportamientos eficaces de RdE (Mestre et al., 2016). Esto apoya la visión de Mayer y Salovey (2007) sobre la idea jerarquizada de la IE. De tal modo que se necesita de un adecuado desarrollo de la percepción precisa de las emociones para después empezar a comprenderlas, y una vez adquirido un buen conocimiento de las mismas estamos en disposición de regularlas y/o gestionarlas.

La vida escolar, el proceso de socialización que implica, da un buen número de oportunidades para desarrollar la capacidad para regular las emociones. El deseo de agradar a los demás, de cumplir con las normas, de generar un buen rendimiento académico, el evitar o disminuir los comportamientos disruptivos, el saber mediar en los conflictos que surgen, en negociar con el profesorado o compañeros y en mejorar la calidad de las relaciones dentro de la comunidad son situaciones que implican una adecuada gestión de las emociones. Facilitar, aconsejar y monitorizar al alumnado a ser protagonista de estos cambios, situaciones o conflictos son oportunidades para el desarrollo de la capacidad clave para un completo desarrollo de la IE, la regulación de emociones. 


\section{Conclusiones}

El hilo fundamental de este trabajo sobre cómo de forma no específica se pueden estimular las capacidades cognitivas implícitas en la IE, no invalida el hecho deseable de qué forma específica se produzcan experiencias y situaciones de fomento de la IE. Sin embargo, sí es una alternativa más plausible, duradera y realista que el profesorado incluya esta denominada información "hot", con significado existencial para las personas como las emociones, en su rol de "desarrollador de capacidades cognitivas en el alumnado.

Hemos visto, que las capacidades descritas en el segundo estrato del modelo CHC como la percepción, el control atencional y el desarrollo del conocimiento pueden explicar satisfactoriamente el desarrollo y expansión de la IE como capacidad (Mestre et al., 2016). Por consiguiente, toda actividad cognitiva escolar que esté unida al desarrollo de dichas capacidades también fomenta, si bien no directamente, el desarrollo de la IE, de ahí, quizás, la conexión entre IE y rendimiento académico con relaciones moderadamente altas (véase Mestre, Guil y Gil-Olarte, 2004).

Del modelo de capacidad de Mayer y Salovey (2007), no consideramos que la segunda rama, denominada facilitación, sea una rama independiente de las otras tres, percepción, comprensión y regulación. Más bien la consideramos implícitamente integrada dentro de las otras tres (MacCann et al., 2014). Por consiguiente, hemos propuesto que para la percepción adecuada y precisa de las emociones se requiere el desarrollo de las capacidades perceptivas (de abajo a arriba) que impliquen un análisis "no filtrado" de estereotipos, prejuicios o creencias irracionales (que sería más una percepción "de arriba abajo"). Siendo las asignaturas de ciencias y ciencias naturales, aunque no las únicas, las más apropiadas para el desarrollo de dicha capacidad perceptiva. Aunque eso no excluye iniciativas que integren la IE con el mindfulness (véase Ramos-Díaz, Enríquez y Recondo, 2012), que creemos acertada, para la mejora de estas capacidades perceptivas.

En cuanto a la comprensión de las emociones, apostamos más por las ciencias sociales y las humanidades, donde la mejora del conocimiento y la conexión con procesos de la información "hot" (como las emociones existenciales) ayudan a mejorar el dominio del entendimiento y de la comprensión, incluida el de las emociones. Tanto para la percepción como la comprensión puede ser estimuladas con buena parte de las asignaturas de la educación obligatoria, sin embargo, creemos que incluyendo la información "hot" en este tipo de actividades escolares (Mayer et al., 2016) se podrían facilitar las capacidades de la IE de una forma más directa.

Por último, la regulación de las emociones implica un mayor número de esfuerzo y de desarrollo cognitivo, incluido el lingüístico, el social, el emocional y el moral; y la vida del centro escolar está llena de oportunidades e interacciones donde el alumnado, asesorado y guiado por la experiencia docente y del orientador/a, podría desarrollar esta capacidad clave para la adaptación personal y social.

Defendemos que el profesorado no puede ser el "elemento solucionador de cada problema, alarma o demanda social"; pero sí estamos convencidos de que el reconocimiento a su figura catalizadora del desarrollo y socialización del alumnado es clave para su papel en el desarrollo de la IE del alumnado. Realizando su 
importante labor como docente instruye al alumnado en el desarrollo de las competencias cognitivas claves e implícitas en la IE, el único esfuerzo extra es añadir información, como hicimos en el caso de la trama de Otelo, que tenga significado para la vida del estudiante. Esta perspectiva de añadir "información hot" a la tarea del docente nos parece más viable y realista, y además conseguimos una mayor temporalidad de dichas influencias en el desarrollo de la IE de los estudiantes. Esperamos, con este artículo, estimular al docente a este "factible" desafío.

\section{REFERENCIAS BIBLIOGRÁFICAS}

Barraca, J., Fernández-González, A. y Sueiro, M. (2009). Test de Sensibilidad a las Interacciones Sociales (TESIS): Una prueba objetiva para la medición de la inteligencia emocional. Bizkaia: AlborCohs.

Belfield, C., Bowden, B., Klapp, A., Levin, H., Shand, R. y Zander, S. (2015). The economic value of social and emotional learning. New York, NY: Center for CostBenefit Studies in Education, Columbia University.

Brackett, M. A. y Rivers, S. E. (2013). Transforming Students' Lives with Social and Emotional Learning. En R. Pekrun y L. Linnenbrink-Garcia (eds.), International Handbook of Emotions in Education (pp. 368-388). New York, NY: Taylor and Francis.

Brackett, M. A., Rivers, S. E., Shiffman, S., Lerner, N. y Salovey, P. (2006). Relating emotional abilities to social functioning: a comparison of self-report and performance measures of emotional intelligence. Journal of Personality and Social Psychology, 91(4), 780-795. DOI: http://doi.org/10.1037/0022-3514.91.4.780.

Bruner, J. S. (1973). The Relevance of Education. New York: Norton.

Detterman, D. K. (1982). Does 'g' exist? Intelligence, 6(2), 99-108. DOI: http://doi. org/10.1016/0160- 2896(82)90008-3.

Duckworth, A. L., Quinn, P. D. yTsukayama, E. (2012). What no child left behind leaves behind: The roles of IQ and self-control in predicting standardized achievement test scores and report card grades. Journal of Educational Psychology, 104(2), 439-451. DOI: http://doi.org/10.1037/a0026280.

Durlak, J. A., Domitrovich, C. E., Weissberg, R. P. y Gullotta, T. P. (eds.). (2015). The Handbook of Social and Emotional Learning. New York, NY: The Guilford Press.

Durlak, J. A., Weissberg, R. P., Dymnicki, A. B., Taylor, R. D. y Schellinger, K. B. (2011). The impact of enhancing students' social and emotional learning: A metaanalysis of school-based universal interventions. Child Development, 82, 405432. Doi:10.1111/j.1467-8624.2010.01564.x.

Flanagan, D. P. y Dixon, S. G. (2013). The Cattell-Horn-Carroll Theory of cognitive abilities. En C. R. Reynolds, K. J. Vannest, y E. Fletcher-Janzen (eds.), Encyclopedia of Special Education (pp. 368-382). Hoboken, NJ: John Wiley \& Sons.

Galotti, K. M. (2008). Cognitive Psychology: In and Out of the Laboratory, 4th ed. Belmont, CA: Thomson Wadsworth.

Goldstein, E. B. (2008). Cognitive Psychology: Connecting Mind, Research, and Everyday Experience, 2nd ed. Belmont, CA: Thomson Wadsworth. 
Gross, J. J. y Barrett, L. F. (2011). Emotion Generation and Emotion regulation: One or Two Depends on Your Point of View. Emotion Review, 3, 1, 8-16. DOI: http://doi. org/10.1177/175407391038097421479078.

Gross, J. J. y Thompson, R. A. (2007). Emotion regulation: Conceptual foundations. En J. J. Gross, (ed.). Handbook of emotion regulation (pp. 3-24). New York: Guilford Press.

Ivcevic, Z. y Brackett, M. A. (2015). Predicting creativity: Interactive effects of openness to experience and emotion regulation ability. Psychology of Aesthetics, Creativity and the Arts, 9(4), 480-487. DOI: http://doi.org/10.1037/a0039826.

Joseph, D. L. y Newman, D. A. (2010). Discriminant Validity of Self-Reported Emotional Intelligence: A Multitrait-Multisource Study. Educational and Psychological Measurement, 70(4), 672-694. DOI: http://doi.org/10.1177/0013164409355700.

Kahneman, D. (2011). Thinking, Fast and Slow. Nueva York: Farrar, Strauss, Giroux.

Kosslyn, S. y Miller, W. (2013). Top brain bottom brain: surprising insights into how you think. Nueva York: Simon and Schuster.

Lazarus, R. S. y Lazarus, B. N. (1996). Passion \& Reason. Nueva York: Oxford University Press.

Legree, P. J., Psotka, J., Robbins, J., Roberts, R. D., Putka, D. J. y Mullins, H. M. (2014). Profile similarity metrics as an alternate framework to score rating-based tests: MSCEIT reanalyses. Intelligence, 47, 159-174. DOI: http://doi.org/10.1016/j. intell.2014.09.005.

Levin, H. M. (2012). More than just test scores. Prospects, 42, 269-284. DOI: http:// doi.org/10.1007/s11125-012-9240-z.

Lopes, P. N., Mestre, J. M., Guil, R., Kremenitzer, J. P. y Salovey, P. (2012). The Role of Knowledge and Skills for Managing Emotions in Adaptation to School: Social Behavior and Misconduct in the Classroom. American Educational Research Journal, 49(4), 710-742. DOI: http://doi.org/10.3102/0002831212443077.

MacCann, C., Joseph, D. L., Newman, D. A. y Roberts, R. D. (2014). Emotional inteIligence is a second-stratum factor of intelligence: Evidence from hierarchical and bifactor models. Emotion, 14(2), 358-74. DOI: http://doi.org/10.1037/a0034755.

Márquez, L. y Mestre, J. M. (2012). Mindfulness: sobre la gestión y evocación de emociones. En C. González-Ferreras, D. González-Manjón, R. Guil y J. M. Mestre (eds.), Aportaciones Recientes al Estudio de la Motivación y de la Emoción (pp. 269-275). Sevilla: Fénix.

Mayer, J. D., Caruso, D. y Salovey, P. (2016). The Ability Model of Emotional Intelligence: Principles and Updates. Emotion Review, 8(4), 290-300. DOI: http://doi. org/10.1177/1754073916639667.

Mayer, J. D. y Salovey, P. (2007). ¿Qué es la inteligencia emocional? En J. M. Mestre y P. Fernández-Berrocal (eds.), Manual de Inteligencia Emocional (pp. 25-45). Madrid: Pirámide.

Mayer, J. D., Salovey, P., Caruso, D. R. y Sitarenios, G. (2003). Measuring emotional intelligence with the MSCEIT V2.0. Emotion, 3(1), 97-105. DOI: http://doi. org/10.1037/1528-3542.3.1.97. 
McGrew, K. S. (2009). CHC theory and the human cognitive abilities project: Standing on the shoulders of the giants of psychometric intelligence research. Intelligence, 37(1), 1-10. DOI: http://doi.org/10.1016/j.intell.2008.08.004.

Medina, R., De La Torre, M. C., Pérez-Alarcón, I., Reyes, M. J., Ceballos-Zúñiga, M. L. y Mestre, J. M. (2012). Una Valoración del TESIS (Test De Sensibilidad De Interacción Social) como Criterio de la Inteligencia Emocional. En C. González-Ferreras, D. González-Manjón, R. Guil y J. M. Mestre (eds.), Aportaciones Recientes al Estudio de la Motivación y de la Emoción (pp. 303-309). Sevilla: Fénix.

Mestre, J. M. (2003). Validación empírica de una escala para medir la inteligencia emocional, desde un modelo mixto, en una muestra de estudiantes de la Bahía de Cádiz. Tesis Doctoral. Universidad de Cádiz.

Mestre, J. M., Araujo, A., Guil, R. y Gil-Olarte, P. (2009). It is enough understanding the anxiety but also managing it. Psychology \& Health, 24, 270-271. DOI: http:// doi.org/10.1080/08870440903126421.

Mestre, J. M., Comunian, A. L. y Comunian, M. L. (2007). Inteligencia emocional: una revisión a sus primeros quince años y un acercamiento conceptual desde los procesos psicológicos. En J. M. Mestre y P. Fernández-Berrocal (eds.), Manual de Inteligencia de inteligencia Emocional (pp. 45-66). Madrid: Pirámide.

Mestre, J. M. y Fernández-Berrocal, P. (2007). Prólogo de los coordinadores. En J. M. Mestre y P. Fernández-Berrocal (eds.), Manual de la Inteligencia Emocional (pp. 21-24). Madrid: Pirámide.

Mestre, J. M. y Guil, R. (2006). Medidas de ejecución versus medidas de autoinformes de Inteligencia Emocional. Ansiedad y Estrés, 12(2-3), 413-425.

Mestre, J. M. y Guil, R. (2012). La Regulación de las Emociones: Una vía para la adaptación personal y social. Madrid: Pirámide.

Mestre, J. M., Guil, R., Brackett, M. y Salovey, P. (2008). Inteligencia Emocional: definición, evaluación y aplicaciones desde el modelo de habilidades de Mayer y Salovey. En F. Palmero y F. Martínez-Sánchez (eds.), Motivación y Emoción (pp. 407-438). Madrid: McGraw-Hill.

Mestre, J. M., Guil, R. y Gil-Olarte, P. (2004). Inteligencia Emocional: algunas respuestas empíricas y su papel en la adaptación escolar en una muestra de alumnos de secundaria. Revista Electrónica de Motivación y Emoción, VII, 6, Recuperado de: http://reme.uji.es/articulos/numero22/article5/numero\%2022\%20article\%20 5\%20INTEL.pdf.

Mestre, J. M., Guil, R., Lopes, P., Salovey, P. y Gil-Olarte, P. (2006). Emotional intelligence and social and academic adaptation to school. Psicothema, 18, 112-118.

Mestre, J. M., Larrán, C., Herrero, J., Guil, R. y de la Torre, G. G. (2015). PERVALE-S: a new cognitive task to assess deaf people's ability to perceive basic and social emotions. Frontiers in Psychology, 6, 1-13. DOI: http://doi.org/10.3389/ fpsyg.2015.01148.

Mestre, J. M., MacCann, C., Guil, R. y Roberts, R. D. (2016). Models of cognitive ability and emotion can better inform contemporary emotional intelligence frameworks. Emotion Review, 8(4), 322-330. DOI: http://doi.org/10.1177/1754073916650497. 
Mestre, J. M., Núñez-Vázquez, I. y Guil, R. (2007). Aspectos psicoevolutivos, psicosociales y diferenciales de la inteligencia emocional. En J. M. Mestre y P. Fernández-Berrocal (eds.), Manual de inteligencia emocional (pp. 151-170). Madrid: Pirámide.

Mestre, J. M., Pérez-Alarcón, I., Larrán, C., Guil, R. y Hidalgo, V. (2014). PERCEXPVAL: Un instrumento para medir la capacidad para percibir y valorar emociones básicas en niños de la etapa infantil (3-6 años). Resultados recientes en su comparación con el Emotion Match Task (EMT). En A. Acosta, J. L. Megías, y J. Lupiáñez (eds.), Avances en el estudio de la Motivación y de la Emoción: VIII Simposio de la Asociación de Motivación y de la Emoción (pp. 181-187). Granada: Universidad de Granada.

Mikolajczak, M. (2009). Moving beyond the ability-trait debate: A three level model of emotional intelligence. E-Journal of Applied Psychology, 5, 25-31.

Nafukho, F. M. (2010). Emotional Intelligence and Performance: Need for Additional Empirical Evidence. Advances in Developing Human Resources, 11(6), 671-689. DOI: http://doi.org/10.1177/1523422309360838.

Nathason, L., Rivers, S., Flynn, L. y Brackett, M. (2016). Creating Emotional InteIligence Schools with RULER. Emotion Review, 8(4), 305-310. DOI: http://doi. org/10.1177/1754073916650495.

Peña-Sarrionandia, A., Mikolajczak, M. y Gross, J. J. (2015). Integrating emotion regulation and emotional intelligence traditions: a meta-analysis. Frontiers Psychology, 6, 160. Doi:10.3389/fpsyg.2015.00160.

Plucker, J. A. y Esping, A. (eds.) (2014). Human intelligence: Historical influences, current controversies, teaching resources. Recuperado de: http://www.intelltheory.com.

Ramos-Díaz, N., Recondo, O. y Henríquez, H. (2012). Practica la Inteligencia Emocional Plena: la gestión eficaz de las emociones a través de mindfulness. Barcelona: Kairós.

Rementería, I. (2001). Prevenir en drogas: paradigmas, conceptos y criterios de intervención. Serie Políticas Sociales. CEPAL/ECLAC. División de Desarrollo Social de Santiago de Chile. Recuperado de: http://www.eclac.org/publicaciones/ xml/4/8044/lcl1596-P.pdf.

Rodrigues Gonzales, A. (1995). La Segunda Guerra Mundial-La Guerra en Europa. Madrid: Akal.

Ruiz-Aranda, D., Cabello, R., Palomera, R., Extremera, N., Salguero, J. M. y Fernández-Berrocal, P. (2013). Programa INTEMO. Guía para mejorar la inteligencia emocional de los adolescentes. Madrid: Pirámide.

Salovey, P. (comp.) (1991). The Psychology of jealousy and envy. Nueva York: Guilford.

Salovey, P., Hsee, C. K. y Mayer, J. D. (1993). Emotional intelligence and the selfregulation of affect. En D. M. Wegner y J. W. Pennebaker (eds.), Emotion regulation in couples and families Pathways to dysfunction and health (pp. 258-277). Englewood Cliffs: Prentice Hall.

Salovey, P. y Mayer, J. D. (1990). Emotional Intelligence. Imagination, Cognition, and Personality, 9(3), 185-211. DOI: http://doi.org/10.1016/S09621849(05)80058-7. 
Schneider, W. J. y Newman, D. A. (2015). Intelligence is multidimensional: Theoretical review and implications of specific cognitive abilities. Human Resource Management Review, 25, 12-27. DOI: http://doi.org/10.1016/j.hrmr.2014.09.004.

Smith, R. H., Kim, S. H. y Parrott, W. G. (1988). Envy and jealousy: Semantic problems and experiential distinctions. Personality and Social Psychology Bulletin, 14, 401-409.

Wranik, T., Barrett, L. F. y Salovey, P. (2007). Intelligent emotion regulation: Is knowledge power? In J. J. Gross (ed.), Handbook of Emotion Regulation (pp. 393407). New York: Guilford Press. 